\title{
Effectiveness of Person-centred Integrated Care for Community-dwelling People With Hypertension and Diabetes in High-income Countries: Protocol for a Systematic Review and Meta-analysis
}

Qiang Tu ( $\nabla$ qiang.tu@sydney.edu.au )

The University of Sydney https://orcid.org/0000-0002-8602-3347

\section{Lily Xiao}

Flinders University College of Nursing and Health Sciences

\section{Karice Hyun}

The University of Sydney School of Health Sciences

Shuanglan Lin

Chongqing Medical University

Jeffrey Fuller

Flinders University College of Nursing and Health Sciences

Nashid Hafiz

The University of Sydney School of Health Sciences

Leila Mohammadi

Flinders University

Julie Redfern

The University of Sydney School of Health Sciences

\section{Protocol}

Keywords: Hypertension, Diabetes, Integrated care, High-income countries, Systematic review

Posted Date: September 15th, 2021

DOl: https://doi.org/10.21203/rs.3.rs-882617/v1

License: (c) (i) This work is licensed under a Creative Commons Attribution 4.0 International License. Read Full License 


\section{Abstract}

Background: Hypertension and diabetes are two of the high prevalent non-communicable diseases. Inadequate care coordination among health systems and between health professionals as well as limited self-care capability of patients lead to the suboptimal care for people with hypertension and diabetes. Person-centred integrated care aiming to address these three levels of barriers has been proposed by World Health Organization as a global strategy to enhance the quality of care for people with chronic diseases. However, the effectiveness and characteristics of integrated care for communitydwelling people with hypertension and diabetes has not been systematically assessed.

Methods: A systematic literature search will be conducted in seven databases including MEDLINE, Emcare, CINAHL, EMBASE, Cochrane Library of systematic reviews, Scopus and ProQuest. Additional studies will be identified through manual searching of reference lists and grey literatures. Quantitative studies (e.g. randomized controlled trials, quasi-randomized trials, before-and-after studies) evaluating the effects of integrated care for community-dwelling people with hypertension and diabetes will be included. Primary outcomes will be systolic and diastolic blood pressure. Secondary outcomes will include the proportion of patients achieving target blood pressure, glycated haemoglobin, fasting blood glucose, blood lipid, treatment adherence, health knowledge, mortality, quality of life, hospitalisation, emergency department use, patient satisfaction, adverse events and caregiver burden. Two reviewers will independently screen articles, extract data and assess study quality. Methodological quality of the selected studies will be assessed using the Revised Cochrane risk-of-bias tool and certainty of evidence using the Grading of Recommendations Assessment, Development and Evaluation (GRADE) system. Disagreements will be resolved through team discussion. Random effects model will be used for data synthesis if feasible.

Discussion: This systematic review will summarise the evidence regarding the overall effectiveness, core components and implementation mechanism of integrated care interventions for people with hypertension and diabetes. Findings from the review will provide crucial evidence to inform the development of health policy, clinical practice and health resources to relieve the burden of hypertension and diabetes on healthcare systems.

Registration: The protocol is registered with PROSPERO (registration number CRD42021271338)

\section{Background}

Hypertension and diabetes are two of the high prevalent non-communicable diseases (NCDs) that have emerged as a global public health crisis (1). It's estimated that 1 billion and 422 million people are living with hypertension and diabetes worldwide, respectively, and this figure is expected to rise greatly over the coming decades $(2,3)$. Hypertension is a common co-morbidity in people with type 2 diabetes, with $74 \%$ having coexisting high blood pressure (4). Patients with hypertension and diabetes often have complex clinical course and multifaceted care needs and are in great need of long-term and a wide range of health 
care services that are well-integrated and person-centred $(5,6)$. However, insufficient care coordination among health systems and between health professionals from different disciplines are widely recognized $(7,8)$. Patients with hypertension and diabetes often experience duplicated, fragmented and uncoordinated care, which inevitably contributes to suboptimal treatment, frequent hospitalization and enormous burden on health systems $(9,10)$.

In order to respond to the challenges associated with chronic diseases and address the complexities of managing multi-morbidities, efforts have been made globally to promote better-integrated care through strengthening the coordination and cooperation among health systems, health professionals, patients and their caregivers $(11,12)$. Person-centred integrated care has been proposed by the World Health Organization as a global strategy to improve health outcomes, enhance quality of patient care and reduce high-cost hospitalization for populations (13).

Integrated care interventions are often multifactorial and complex, which can take place across multiple levels. These are the macro level of health systems that enhance collaboration between health organizations, the meso level of health professionals that facilitates multi-disciplinary team in delivery of care services, and the micro level of individuals that promotes multi-component self-care $(14,15)$. Despite the importance of implementing multi-level and multi-component interventions, actual practices are often limited across these three levels leading to suboptimal integration of health services, poor health outcomes and unsatisfactory patient experiences $(13,16)$. A factor limiting the implementation of integrated care is the lack of a taxonomy of the necessary elements that reflects its inherent multi-level and multi-component nature (16). Therefore, transforming towards integrated care requires a comprehensive understanding of the requisite elements and mechanism of this care approcah, which calls for a systematic review.

Varied and inconsistent definitions of integrated care $(8,17)$ and failure to recognise the inherent natures of these elements have led to mixed results and difficulties in developing and scaling up integrated care (18). The heterogeneous nature of integrated care intervention components resulting from conceptual ambiguity hinders meta-analysis for pooled effect size and poses challenges to the replicability and reliability of studies $(10,19)$. A systematic review by Baxter et al. (2018) evaluated the efficiency of integrated care on service delivery in terms of access to care and quality of care (19). The lack of a precise pre-specified definition of integrated care and disease-defined patient group precluded metaanalysis of clinical outcomes. Similarly, systemetic reviews by Liljas et al. (2019) and Martínez-González et al. (2014) identifing the elements of effective integrated care in improving health outcomes $(8,20)$ solely examined the organization (macro) level and did not take into account the scale of the interventions at micro and meso levels. Hence, to understand the highly complex nature of integrated care, existing research needs to be examined using a clear classification of the components supported by a specific theoretic framework.

To enable comparison of the studies included in the review, we have used the definition of integrated care as proposed by the World Health Organisation and by Rainbow Model for Integrated Care. 
"Integrated care is an approach to strengthen people-centred health systems through the promotion of the comprehensive delivery of quality services across the life-course, designed according to the multidimensional needs of the population and the individual and delivered by a coordinated multidisciplinary team of providers working across settings and levels of care"(13).

The Rainbow Model for Integrated Care has emphasized that effective and well-developed person-centred integrated care should be based on multi-level multiple-component care integration that includes wellorganised health systems, individually-tailed patient support and close engagement of health professionals (21).

The scope of integrated care varies across high- income countries (HICS) to low- and- middle- income countries (LMICs). Given considerable context-specific variations of integration mechanisms in integrated care, it is important to evaluate integrated care in a specific context and populations. To reduce the impacts from clinical heterogeneity in terms of population and settings, our study only focuses on the review of integrated care in high-income countries.

To our knowledge, only two systematic reviews have examined integrated care for diabetes pertaining to intervention types and outcomes $(22,23)$. The review by Busetto et al. (2016) was restricted to only two databases, which possibly led to a large number of relevant studies being overlooked and therefore the summarised evidence inconclusive (22). The other review by Lim et al. (2018) only included integrated care interventions that lasted at least 12 months, which may lead to a high risk of search bias (23). The definitive impact of disease-specific integrated care for both hypertension and diabetes remains unclear. This review addresses the knowledge gap in the literatures.

To date, research evidence is lacking on how to best design, organize and deliver integrated care for people with hypertension and diabetes and the extent to which full care integration (the combinations of all three levels of care integration) improves health outcomes compared with partial care integration. Given the variability in terms of intervention ingredients, duration, delivery process and disease type, there is a need to undertake a systematic review to explore the components and effectiveness of interventional studies to improve person-centred of integrated care for people with hypertension and diabetes in highincome countries.

\section{Aims}

The primary aim of this review is to systematically evaluate the effectiveness of person-centred integrated care interventions for people with coexisting hypertension and diabetes. We also aim to identify the key characteristics, core components and delivery methods of effective integrated care strategies through analysis of integrated care across macro, meso and micro levels. The PICO (Population, Intervention, Comparison, Outcomes and Study designs) framework of the Cochrane review will formulate eligibility criteria of studies and ensure search quality (24). 


\section{Methods}

The study has been registered in the International Prospective Register of Systematic Reviews (PROSPERO) database (CRD42021271338) and is reported in accordance with the Preferred Reporting Items for Systematic Reviews and Meta-Analyses Protocols (PRISMA-P) statement (25) (see Additional file 1). The proposed systematic review and meta-analysis will be conducted following the guidance of the Cochrane Collaboration Handbook of Systematic Reviews. Any deviations from the protocol will be updated in the PROSPERO record and the final published manuscript.

\section{Included studies}

\section{Study Design}

The review will include randomized controlled trials (RCTs) including cluster RCTs, controlled clinical trials (CCTs), quasi RCTs and before-and-after studies to evaluate the effects of the integrated care. Other types of studies including cross-sectional studies, qualitative studies, cohort studies, study protocols, abstracts and review articles will be excluded.

\section{Participants}

Studies will be included if the participants: (1) are aged > 18 years; (2) have multimorbidity of hypertension and type 2 diabetes; (3) live in the community or primary care settings; (4) come from HICs. We will exclude those studies if the participants: (1) have an undiagnosed hypertension or type 2 diabetes; (2) have type 1 diabetes or gestational diabetes or prediabetes or prehypertension; (3) have a serious mental illness or cognitive impairment; (4) are not living in the community or primary care settings; (5) come from LMICs.

\section{Interventions}

For the purpose of this review, the concept of integrated care is linked to the validated and universally accepted Rainbow Model for Integrated Care, which postulates that improving integrated care requires changes in three interdependent aspects: organizational (macro), health professionals (meso), and individual (micro) levels. Hence, this review will include person-centred and integrated care interventions for people with hypertension and diabetes through integration of care services at these three levels. Given that integrated care can target multiple levels of service provision, studies comprising at least one of the following types of care integration will be included in the review. 
Table 1

Intervention types and components

1. Interventions aimed at integrating and coordinating care among different health care facilities or units (Macro - organization-level care integration)
1.1 Collaboration and communication between different levels of care setting (primary, secondary and tertiary care settings)

1.2 Referral to other health care facilities for further treatment

1.3 Organizational changes or redesign of a delivery system
2. Interventions aimed at integrating and coordinating care among different health care professionals (Meso health professionals-level care integration)

2.1 Multi-disciplinary team (GPs, community nurses, case managers, dieticians, pharmacists, social workers, psychologists, etc.)

2.2 Revision of professional roles or staff relocation

\subsection{Case management}

3. Interventions aimed at integrating and coordinating care at individual patient level (Micro - individual-level care integration)
3.1 Multi-component self-care support (health education, lifestyle modifications, motivational support, treatment adherence, goal setting, etc.)

3.2 Involving people and/or their caregivers in self-care support, decision making and care planning, by taking individual's care needs into account

\subsection{Personal home care plan}

3.4 Peer support

Comparisons will be made with routine community care, or usual clinical care, or conventional care provided in the community setting or home.

\section{Outcome measures}

The primary outcome measures will be systolic and diastolic blood pressure.

Secondary outcomes cover the following four categories:

(1) patient clinical outcomes: the proportion of patients achieving target blood pressure; glycated haemoglobin (HbA1c); fasting blood glucose; blood lipid, mortality, adverse events; (2) patient-reported outcomes: health knowledge; health-related quality of life; treatment adherence, patient satisfaction; (3) use of healthcare resources: hospitalisation, emergency department use; and (4) health professionals outcomes: health professionals' satisfaction, caregiver burdens. 
A systematic search of literature will be undertaken in seven electronic databases that include MEDLINE, Emcare, CINAHL, EMBASE, Cochrane Database of Systematic Reviews, Scopus and ProQuest from 1995 and onwards. Restricting the literature search from 1995 and onwards is because few studies regarding integrated care were published before 1995 (8). The retrieval of citations will be restricted to published studies in the English languages. To ensure a wider search and minimize the publication bias due to electronic searching, other searching techniques will also be employed, including manual search of reference lists of primary studies, grey literature searches (e.g. conference papers, thesis, reports), as well as backward and forward citation searches. References and abstracts retrieved through the systematic searches will be exported to and managed by Endnote X9 Referencing Software. The searching procedure and reasons for study exclusion will be reported in accordance with the PRISMA (Preferred Reporting Items for Systematic Review and Meta-analysis) guideline. A detailed search strategy for MEDLINE developed in consultation with a medical librarian is presented in Additional file 2. Similar search strategy will be adapted to other databases.

\section{Study screening and selection}

Study screening will be independently performed by two reviewers through a two-stage selection process. In the first stage, after duplicates are removed, titles and abstracts will be independently screened based on pre-designed inclusion criteria. Eligible studies will be included for full-text review. In the second stage, the full text of selected studies will be retrieved and critically assessed by the same two independent reviewers to further determine the eligibility of studies. Any discrepancy will be resolved through team discussion until consensus is reached. The reasons for excluding the studies assessed in full will be recorded and presented in the final manuscript. The process of study screening and selection will be documented using the PRISMA flow diagram.

\section{Data extraction}

Two reviewers will independently extract and verify the data from the included studies using a standardized Cochrane data extraction sheet for intervention reviews. The extracted data will include (1) study information (authors, year of publication, country of origin); (2) participant characteristics (e.g. sample size, age, gender, ethnicity, comorbidities); (3) Intervention and comparator characteristics (role and type of healthcare professionals, level of care integration, content and components of interventions, theoretical framework, mode of intervention delivery (frequency, duration, treatment fidelity), and implementation strategies); (4) study characteristics (study setting, design and methods); (5) outcome measures (primary and secondary measures, timing and frequency of assessments, tool used); and (7) main findings (statistically significant change, effect size). Data on repeated measures of these outcomes from pre-intervention to short-term follow-up (3 months), mid-term follow-up (6 months) and long-term follow-up (12 months) will be extracted for pooled effect analyses. The authors of studies will be contacted if there are any missing information or unclear data.

\section{Appraisal of risk of bias and strength of evidence}


Studies fulfilling eligibility criteria will be independently evaluated for methodological quality and risk of bias by two reviewers using the latest Revised Cochrane risk-of-bias tool for randomized trials (RoB 2) and ROBINS-I tool for non-randomised studies (ROBINS-I) (26-28). RoB 2 assesses the overall risk of bias based on the considerations of five distinct domains: randomisation process; deviations from intended interventions; missing outcome data; measurement of the outcome; selection of the reported result. Each domain will be ranked as "low risk", "some concerns" or "high risk" based on the information extracted from studies. Overall assessment of the quality of each study will be undertaken based on the judgement of all domains. ROBINS-I assesses bias due to confounding, selection of participants, intervention classification, deviations from intended interventions, missing data, measurement of outcomes, selection of reported results, and overall bias. The ratings for each bias domain will be judged as low, moderate, serious or critical risk (28).

To reduce the potential misinterpretation of the findings, strength of evidence for each outcome will be assessed using the Grading of Recommendations Assessment, Development and Evaluation (GRADE) system (29). The quality of evidence will be classified into four levels: high, moderate, low and very low quality, according to the judgment on five domains (limitations of design, inconsistency, indirectness, imprecision and other factors). The certainty of evidence will be independently rated by two reviewers. Discrepancies will be resolved by team discussion until consensus is reached.

\section{Data analysis and synthesis}

Meta-analysis will be conducted to estimate pooled effect of the intervention on outcomes using Comprehensive Meta-Analysis Software version 3.0 if sufficient data is available in at least three studies retained. To enable pooling and comparison of the outcomes measured by different scales, the pooled standardized mean difference with $95 \%$ confidence interval $(\mathrm{Cl})$ will be calculated as effect size for continuous outcomes (30). For dichotomous outcomes, risk ratio (RR) with $95 \% \mathrm{Cl}$ will be calculated as effect size. Heterogeneity in intervention effects between studies will be quantified using $\mathrm{I}^{2}$ statistic. $\left.\right|^{2}$ values of $25 \%, 50 \%$ and $75 \%$ will indicate low, moderate and high levels of heterogeneity, respectively (31). There is likely to have heterogeneity in the type of intervention, delivery and study design. To mitigate the impacts of clinical or methodological heterogeneity, random-effects model will be used to estimate the pooled effect size (32). Cohen's $d$ will be used to report the effect sizes, where 0.2 represents a small effect, 0.5 a moderate effect and 0.8 a large effect (33). The pooled effect for each outcome will be presented in the form of forest plot. Publication bias will be evaluated by visual examination of funnel plots and Egger's regression tests. To determine the robustness of outcomes and improve generalizability, sensitivity analysis will be undertaken by excluding studies with high risk of bias. A narrative synthesis will be performed if quantitative synthesis is deemed inappropriate due to significant statistical, clinical or methodological heterogeneity. The reporting of narrative synthesis will be guided by synthesis without meta-analysis guideline when meta-analysis is not feasible (34).

To investigate sources of heterogeneity, subgroup analysis will be performed if there is significant variability across studies (35). In addition, to achieve the study objectives that explore the components 
and implementation mechanism associated with effective integrated care, we will also conduct subgroup analysis based on the following intervention characteristics if there are sufficient data available.

i. Different types of care integration (e.organizational level integration vs individual level integration).

ii. Combination of different integration types (e.multiple levels of care integration vs single level care integration).

iii. Intensity of integrated care (e.high-intensity integrated care interventions (more frequent vs lowintensity integrated care interventions (less frequent interventions).

iv. Health professionals implementing integrated care (e.multi-disciplinary team-led vs nurse-led).

v. Length and duration of integrated care (e.the timeframe of follow-up: short-term follow-up (3 vs longterm follow-up (12 months or more).

\section{Discussion}

There is currently little evidence concerning the benefits of integrated care on health outcomes for people with hypertension and diabetes in high-income countries. To our knowledge, the proposed systematic review will be the first to identify, synthesize and assess the best available evidence on the effectiveness of the integrated care for hypertension and diabetes management among people in high-income countries. Further, there is a lack of clarity on which key components of the integrated care are essentially associated with positive health outcomes for people with hypertension and diabetes. Highlighting the core components of integrated care developed specifically for people with hypertension and diabetes will thus be of value to the quality improvement of hypertension and diabetes management and the translation of this research to clinical practice and improve health outcomes for this patient population.

The novelty of the current systematic review is that it examines the effects of integrated care in a more comprehensive way, across the three micro, meso and macro levels. Undertaking comparative analysis of the efficacy of different levels of care integration on health outcomes for people with hypertension and diabetes will guide provide empirical evidence in implementation strategies of hypertension and diabetes healthcare services and guide the future design of translational research in this area. Consequently, findings from this proposed systematic review will inform policy-makers and health providers on how to effectively organize, coordinate and deliver customized interventions in an integrated way and provide insights into remodelling of integrated care that is tailored to the needs of this clinical population. It is anticipated that the study findings will direct fragmented care model in those HICs towards improvements in an integrated and sustainable health service delivery that better meets the needs of patients. The evidence generated from this review will also have the potential of functioning as a useful reference to the management of other chronic diseases. Study findings will be disseminated via peerreviewed publications and presentations at an international conference.

\section{Abbreviations}


CCTs: Controlled clinical trials; CINAHL: Cumulative Index for Nursing and Allied Health Literature; HICs: High- income countries; LMICs: Low- and middle- income countries; PRISMA-P: Preferred Reporting Items for Systematic Reviews and Meta-Analyses Protocols; PROSPERO: International Prospective Register of Systematic Reviews; RCTs: Randomized controlled trials; SMD: Standard mean difference;

\section{Declarations}

\section{ETHICS APPROVAL AND CONSENT TO PARTICIPATE}

Not applicable

\section{CONSENT FOR PUBLICATION}

Not applicable

\section{AVAILABILITY OF DATA AND MATERIALS}

The study protocol does not include the dataset.

\section{COMPETING INTERESTS}

The authors declare that they have no competing interests.

\section{FUNDING}

No specific funding has been received for this systematic review. However, KH's salary is funded by a National Health and Medical Research Council Investigator Grant [GNT1196724] and JR's salary is funded by a National Health and Medical Research Council Career Development Fellowship [GNT1143538].

\section{AUTHORS' CONTRIBUTIONS}

QT conceived and designed the study. All authors contributed to the development of this protocol. QT wrote the first draft of the manuscript. LM (librarian) provided support in developing the search strategy. All authors read, provided feedback, and approved the final manuscript. KH will help data analysis. QT and SL will screen the databases and extract data. The guarantor of the review will be QT.

\section{ACKNOWLEDGEMENTS}

Not applicable

\section{References}

1. Mohan V, Seedat YK, Pradeepa R. The Rising Burden of Diabetes and Hypertension in Southeast Asian and African Regions: Need for Effective Strategies for Prevention and Control in Primary 
Health Care Settings. International Journal of Hypertension. 2013;2013:409083.

2. World Health Organisation. Diabetes Genenva2021 [Available from: https://www.who.int/healthtopics/diabetes\#tab=tab_1.

3. World Health Organisation. Hypertension Geneva2019 [Available from: https://www.who.int/newsroom/fact-sheets/detail/hypertension.

4. Passarella P, Kiseleva TA, Valeeva FV, Gosmanov AR. Hypertension Management in Diabetes: 2018 Update. Diabetes Spectrum. 2018;31(3):218.

5. van der Heide I, Snoeijs S, Quattrini S, Struckmann V, Hujala A, Schellevis F, et al. Patientcenteredness of integrated care programs for people with multimorbidity. Results from the European ICARE4EU project. Health Policy. 2018;122(1):36-43.

6. Busse R, Stahl J. Integrated Care Experiences And Outcomes In Germany, The Netherlands, And England. Health Affairs. 2014;33(9):1549-58.

7. Matthys $E$, Remmen R, Van Bogaert P. An overview of systematic reviews on the collaboration between physicians and nurses and the impact on patient outcomes: what can we learn in primary care? BMC Family Practice. 2017;18(1):110.

8. Liljas AEM, Brattström F, Burström B, Schön P, Agerholm J. Impact of Integrated Care on PatientRelated Outcomes Among Older People - A Systematic Review. International journal of integrated care. 2019;19(3):6.

9. Damery S, Flanagan S, Combes G. Does integrated care reduce hospital activity for patients with chronic diseases? An umbrella review of systematic reviews. BMJ Open. 2016;6(11):e011952.

10. Flanagan S, Damery S, Combes $G$. The effectiveness of integrated care interventions in improving patient quality of life (QoL) for patients with chronic conditions. An overview of the systematic review evidence. Health Qual Life Outcomes. 2017;15(1):188-

11. Kearney LK, Zeiss AM, McCabe MA, Thistlethwaite JE, Chana N, Chen S, et al. Global approaches to integrated care: Best practices and ongoing innovation. The American psychologist. 2019.

12. Desmedt M, Vertriest S, Hellings J, Bergs J, Dessers E, Vankrunkelsven P, et al. Economic Impact of Integrated Care Models for Patients with Chronic Diseases: A Systematic Review. Value Health. 2016;19(6):892-902.

13. World Health Organisation. Integrated care models: an overview. 2016.

14. Angus L, Valentijn PP. From micro to macro: assessing implementation of integrated care in Australia. Australian journal of primary health. 2018;24(1):59-65.

15. Deschodt M, Laurent G, Cornelissen L, Yip O, Zúñiga F, Denhaerynck K, et al. Core components and impact of nurse-led integrated care models for home-dwelling older people: A systematic review and meta-analysis. Int J Nurs Stud. 2020;105:103552.

16. Briggs AM, Valentijn PP, Thiyagarajan JA, Araujo de Carvalho I. Elements of integrated care approaches for older people: a review of reviews. BMJ open. 2018;8(4):e021194-e.

17. Goodwin N. Understanding Integrated Care. International journal of integrated care. 2016;16(4):6-. 
18. Sadler E, Potterton V, Anderson R, Khadjesari Z, Sheehan K, Butt F, et al. Service user, carer and provider perspectives on integrated care for older people with frailty, and factors perceived to facilitate and hinder implementation: A systematic review and narrative synthesis. PLOS ONE. 2019;14(5):e0216488.

19. Baxter S, Johnson M, Chambers D, Sutton A, Goyder E, Booth A. The effects of integrated care: a systematic review of UK and international evidence. BMC Health Services Research. 2018;18(1):350.

20. Martínez-González NA, Berchtold P, Ullman K, Busato A, Egger M. Integrated care programmes for adults with chronic conditions: a meta-review. Int J Qual Health Care. 2014;26(5):561-70.

21. Milawaty N, Pim PV, Mary Ann CB, Yee Wei L, Hubertus Jm V. A Prospective Validation Study of a Rainbow Model of Integrated Care Measurement Tool in Singapore. International journal of integrated care. 2016;16(1).

22. Busetto L, Luijkx KG, Elissen AM, Vrijhoef HJ. Intervention types and outcomes of integrated care for diabetes mellitus type 2: a systematic review. J Eval Clin Pract. 2016;22(3):299-310.

23. Lim LL, Lau ESH, Kong APS, Davies MJ, Levitt NS, Eliasson B, et al. Aspects of Multicomponent Integrated Care Promote Sustained Improvement in Surrogate Clinical Outcomes: A Systematic Review and Meta-analysis. Diabetes Care. 2018;41(6):1312-20.

24. Eriksen MB, Frandsen TF. The impact of patient, intervention, comparison, outcome (PICO) as a search strategy tool on literature search quality: a systematic review. J Med Libr Assoc. 2018;106(4):420-31.

25. Shamseer L, Moher D, Clarke M, Ghersi D, Liberati A, Petticrew M, et al. Preferred reporting items for systematic review and meta-analysis protocols (PRISMA-P) 2015: elaboration and explanation. BMJ : British Medical Journal. 2015;349:g7647.

26. Higgins J, Green S. Cochrane handbook for systematic reviews of interventions. Version 5.1.0 The Cochrane Collaboration; 2011.

27. Sterne JAC, Savović J, Page MJ, Elbers RG, Blencowe NS, Boutron I, et al. RoB 2: a revised tool for assessing risk of bias in randomised trials. BMJ. 2019;366:14898.

28. Schünemann HJ, Cuello C, Akl EA, Mustafa RA, Meerpohl JJ, Thayer K, et al. GRADE guidelines: 18. How ROBINS-I and other tools to assess risk of bias in nonrandomized studies should be used to rate the certainty of a body of evidence. J Clin Epidemiol. 2019;111:105-14.

29. Granholm A, Alhazzani W, Møller MH. Use of the GRADE approach in systematic reviews and guidelines. Br J Anaesth. 2019;123(5):554-9.

30. Sawilowsky S. New Effect Size Rules of Thumb. Journal of Modern Applied Statistical Methods. 2009;8(2):597-9.

31. Melsen WG, Bootsma MCJ, Rovers MM, Bonten MJM. The effects of clinical and statistical heterogeneity on the predictive values of results from meta-analyses. Clinical Microbiology and Infection. 2014;20(2):123-9.

32. Borenstein M, Hedges LV, Higgins JP, Rothstein HR. A basic introduction to fixed-effect and randomeffects models for meta-analysis. Research synthesis methods. 2010;1(2):97-111. 
33. Sullivan GM, Feinn R. Using Effect Size-or Why the P Value Is Not Enough. J Grad Med Educ. 2012;4(3):279-82.

34. Campbell M, McKenzie JE, Sowden A, Katikireddi SV, Brennan SE, Ellis S, et al. Synthesis without meta-analysis (SWiM) in systematic reviews: reporting guideline. BMJ. 2020;368:16890.

35. Richardson M, Garner P, Donegan S. Interpretation of subgroup analyses in systematic reviews: A tutorial. Clinical Epidemiology and Global Health. 2019;7(2):192-8.

\section{Supplementary Files}

This is a list of supplementary files associated with this preprint. Click to download.

- Additionalfile1.docx

- Additionalfile2.docx 